\title{
Marcadores Moleculares y Biomarcadores de Imagen. Importancia en el Avance Terapéutico de los Tumores Cerebrales Intraaxiales
}

\author{
Molecular Markers and Image Biomarkers. Importance \\ in the Therapeutic Advance of Intraaxial Brain Tumors
}

Ulises Guajardo-Hernández ${ }^{1}$; Federico Geisse Martínez ${ }^{2}$ 'Luis Martí-Bonmatí;
Roger Gejman Enriquez ${ }^{4}$ \& David Rojas Zalazar

GUAJARDO-HERNÁNDEZ, U.; GEISSE, M. F.; MARTÍ-BONMATÍ, L.; GEJMAN. E. R. \& ROJAS, Z. D. Marcadores moleculares y biomarcadores de imagen. Importancia en el avance terapéutico de los tumores cerebrales intraaxiales. Int. J. Morphol., 39(2):601-606, 2021.

RESUMEN: La clasificación de los Tumores Primarios del Sistema Nervioso Central (SNC) tiene su origen en la descripción morfológica, cuyo análisis histopatológico ha permitido identificar la línea celular involucrada en estos tumores y obtener el reconocimiento de ciertas características de estas lesiones y su evolución clínica. El estudio molecular ha venido a complementar el diagnóstico inicial permitiendo reconocer entidades que no son distinguibles de otra manera y que han variado los conceptos y definiciones de varias entidades patológicas que modifican el horizonte visible de estas enfermedades. El papel de las imágenes de Resonancia Magnética (RM) en el manejo de los tumores intraaxiales se puede dividir ampliamente en el diagnóstico y la clasificación de los tumores, la planificación del tratamiento y el tratamiento posterior. El presente artículo resume la evidencia epidemiológica relacionada en la clasificación de los tumores primarios del SNC con marcadores moleculares y biomarcadores de imágenes de RM, apuntando a la importancia del uso de la investigación clínica con el manejo terapéutico.

PALABRAS CLAVE: Tumor cerebral; Imágenes RM; Biomarcadores.

\section{INTRODUCCIÓN}

La clasificación de los tumores primarios del Sistema Nervioso Central (SNC) tiene su origen en la descripción morfológica cuyo análisis histopatológico ha permitido describir la estirpe celular involucrada en estas neoplasias y obtener el reconocimiento de ciertas características propias de estas lesiones y su evolución clínica. (Fig. 1) No obstante, la sola descripción histopatológica presenta dificultades para resolver la complejidad y singularidad de la evolución clínica de cada paciente y su manejo terapéutico.

La caracterización según líneas celulares ha ido demostrando incertidumbre en los patrones de presentación y respuesta a la terapia que se resumen en (Cuevas et al., 2011):

- Variabilidad en el diagnóstico histopatológico (inter-observador, inter-sesión, inter-centro).
- Variabilidad en la evolución clínica independiente del tratamiento efectuado.

Con los avances en aspectos bioquímicos y estudios genéticos se ha accedido progresivamente a la obtención de características físico-químicas y moleculares que permiten obtener un conocimiento cada vez mayor respecto de cómo las células patológicas en estas neoplasias alteran cadenas metabólicas fuera del control normal del organismo atentando contra la integridad de este.

El estudio molecular ha venido a complementar el diagnóstico inicial, permitiendo reconocer entidades indistinguibles de otro modo y redefinir los conceptos y las expresiones de diversas entidades patológicas, modificando el horizonte visible de estas enfermedades.

\footnotetext{
${ }^{1}$ Departamento de Especialidades Médicas. Universidad de La Frontera. Temuco Chile.

${ }^{2}$ Residente de Neurocirugía, Universidad de La Frontera. Temuco Chile.

${ }^{3}$ Director Área Clínica de Imagen Médica y del Grupo de Investigación Biomédica en Imagen del Hospital Universitario y Politécnico La Fe, Valencia España.

${ }^{4}$ Jefe de Servicio Anatomía Patológica. Hospital Universidad Católica de Chile. Santiago Chile.

${ }^{5}$ Director Departamento de Ciencias Neurológicas. Universidad de Chile. Santiago Chile.
} 


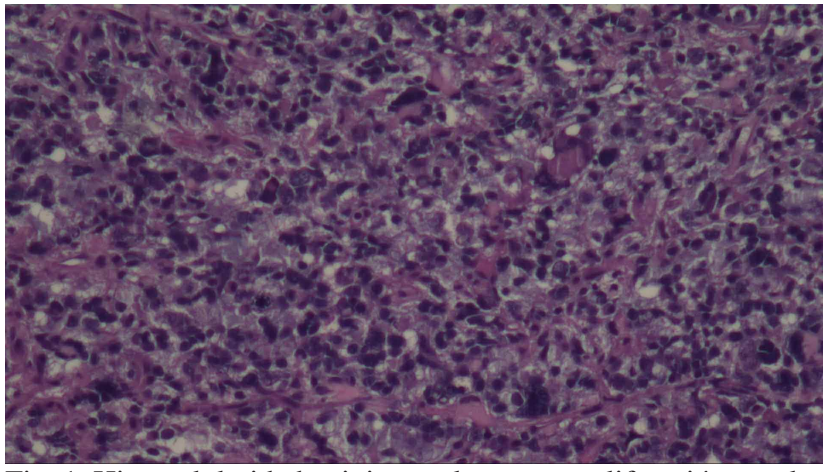

Fig. 1. Hipercelularidad, atipias nucleares y proliferación vasclar visibles en imagen histopatológica (HE) de un glioblastona multiforme.

Por su parte, el estudio con imágenes ha ido también avanzando progresivamente desde el reconocimiento de las estructuras macroscópicas afectados (Fig. 2 a, b) y las principales características tisulares de las lesiones, hasta alcanzar la expresión funcional (Figs. 3 y 4 )de estas con el reconocimiento de la irrigación tisular, con sus componentes vascular y de permeabilidad, y sus componentes físico-químicos moleculares, que da cuenta de las cadenas metabólicas comprometidas.

Una rama de la Medicina, clave para el manejo diagnóstico y terapéutico de esta patología oncológica, es la Neurocirugía, la que ha ido progresando sustancialmente en diversos ámbitos de su ejercicio, particularmente en lo que respecta al conocimiento anatómico y funcional para desarrollar técnicas más seguras, mejorando la exéresis macroscópica. No obstante, el pronóstico y los resultados generales distan mucho de ser satisfactorios.

El reconocimiento actual de la complejidad de esta patología plantea posibilidades de tratamiento propias de la Medicina Personalizada, debiendo considerarse el reconocimiento de un tumor específico dentro de una gama de tumores cuyas características intrínsecas, tanto macroscópicas, de localización y compromiso particular en un determinado lugar del SNC, y caracterizado por diversos marcadores moleculares, lo hacen único y obliga a un tratamiento singular y específico.

El acceso al conocimiento otorgado por las técnicas de neuroimagen ha determinado un avance sustancial al incorporar a la evaluación de la morfología convencional el conocimiento de la funcionalidad tisular y los estudios moleculares para así acercarse a una progresiva mayor comprensión del funcionamiento de los circuitos metabólicos y fisiopatológicos de estos tumores. Esta nueva información se pretende sea de interés para planificar intervenciones más eficientes a través de la farmacología, inmunoterapia, ra-
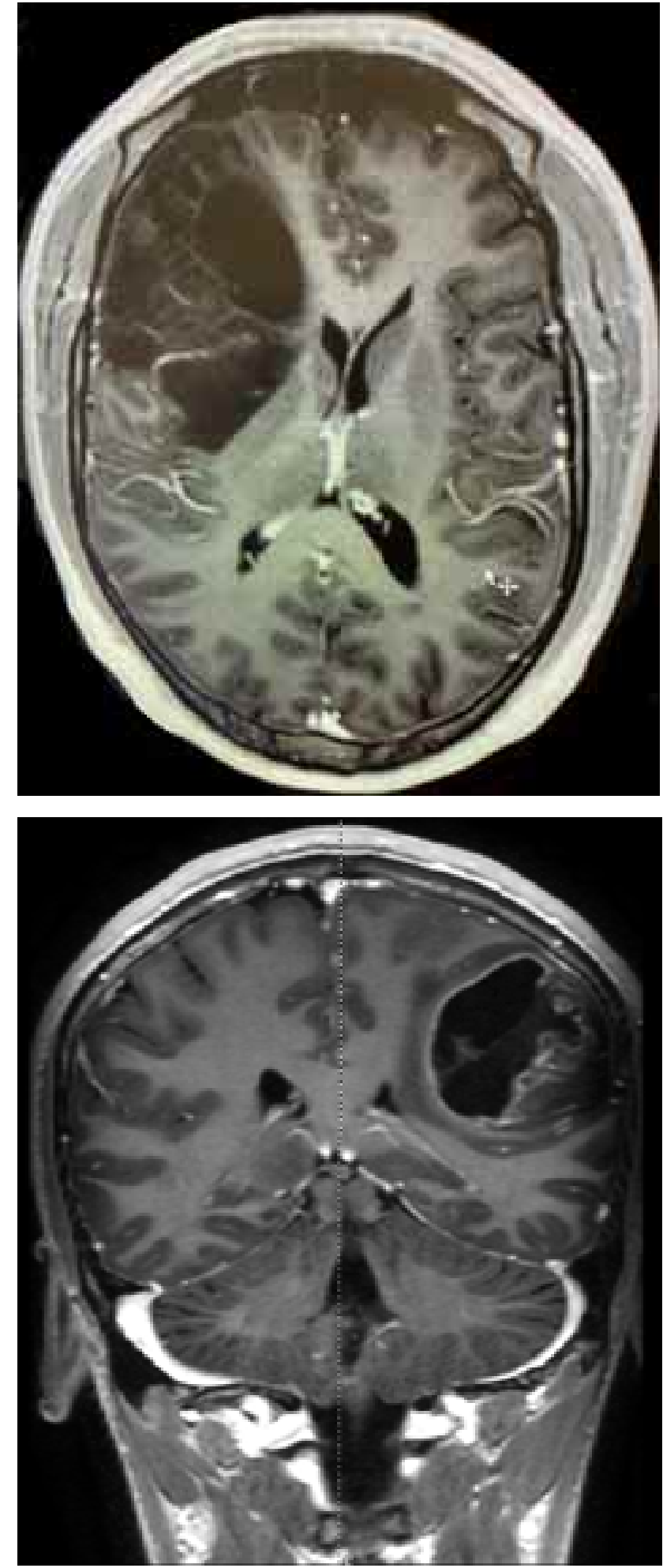

Fig. 2 a y b . Tomografía computada en cortes axiales y coronales de glioma anaplásico y glioblastoma multiforme.

dioterapia, neurocirugía y otras técnicas, lo que aumentará el poder y la eficacia para afectar y controlar su biología. 


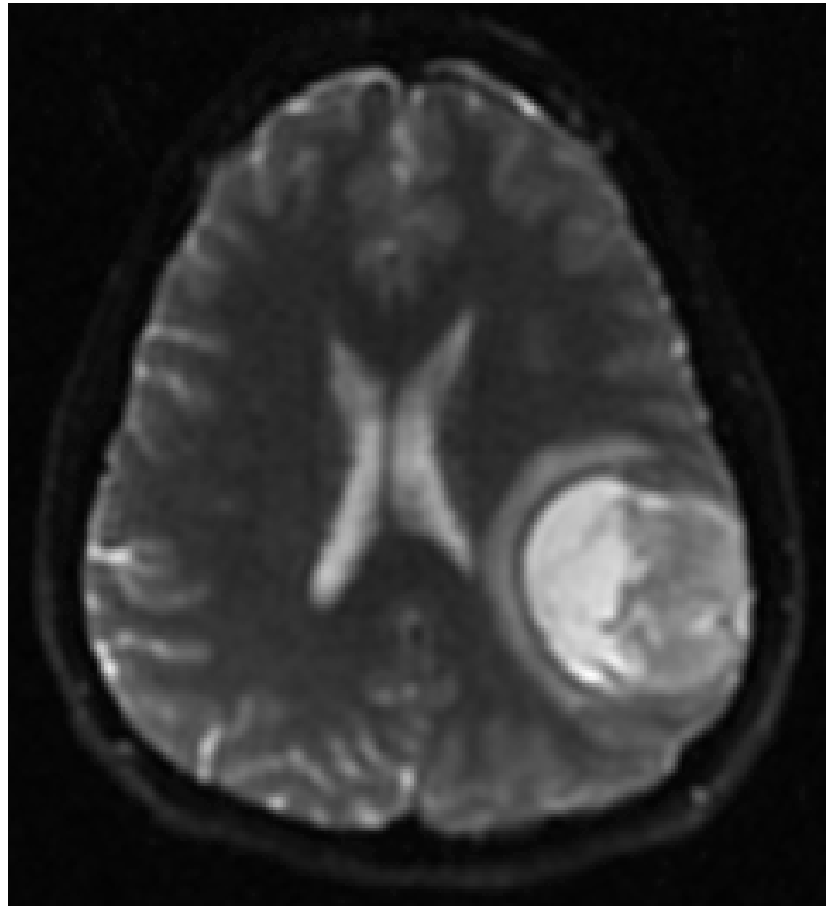

Fig. 3. Glioblastoma multiforme. Secuencia de difusión.

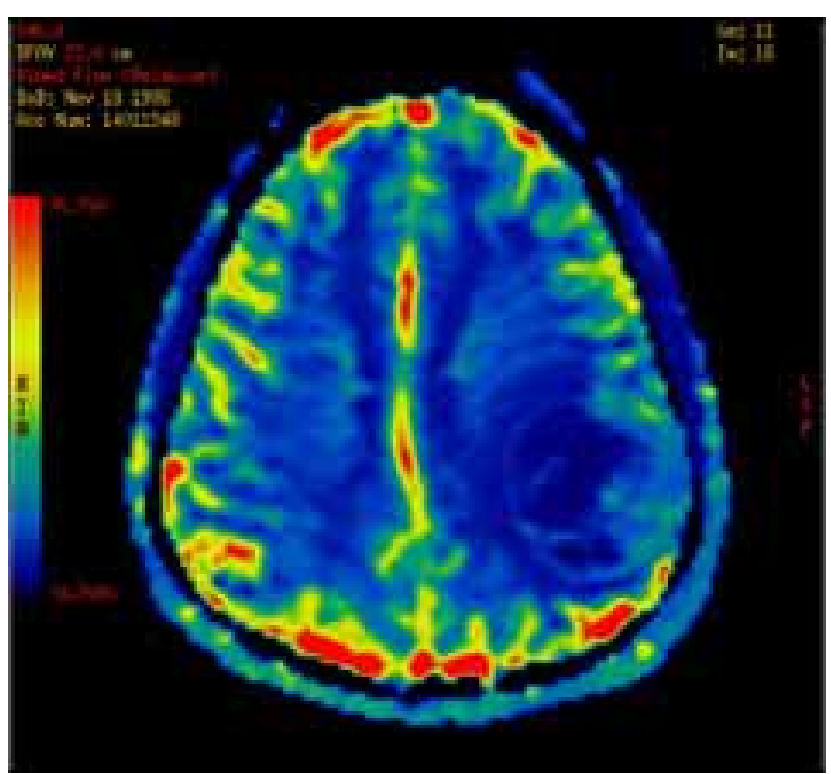

Fig. 4. Glioblastoma multiforme. Secuencia de perfusión.

\section{DATOS EPIDEMIOLÓGICOS RELEVANTES}

Entre 2011-2015 en Estados Unidos los tumores cerebrales del SNC (malignos y no malignos) en personas de 15 a 39 años fueron el segundo tipo de neoplasia más común en hombres y el tercer tipo en mujeres. En personas mayores de 40 años fueron el octavo más común en hombres y el quinto en mujeres (Ostrom et al., 2018). Según el Global Cancer Observatory (Globocan) las estima- ciones indican que en la región de América Central y del Sur, los tumores del SNC fueron la causa número $11 \mathrm{de}$ morbilidad por neoplasia y mortalidad en 2012 (Ferlay, 2015).

\section{CLASIFICACIÓN}

Ya en 1863, Virchow publicó su primer informe de tumores cerebrales. Posteriormente, en 1979, Zülch et al. (1979) desarrollaron una nueva clasificación que fue adoptada por la Organización Mundial de la Salud (OMS). La clasificación de la OMS asigna un grado tumoral (del grado I al grado IV) según su comportamiento clínico y patológico (Kleihues, 2002). Esta clasificación se ha ido actualizando en 1993, 2000, 2007 y, con la utilización de biomarcadores moleculares específicos, se modificó el 2016 (Louis et al., 2007); (Toro-Moreno et al., 2017). La determinación del tipo y grado del tumor como expresión de la agresividad tumoral a partir sólo del estudio histológico tiene una pobre concordancia inter e intra observador (36-62 \% y 51-74 \%, respectivamente) (Mittler, et al., 1996). A diferencia de otros tipos de cáncer, los tumores primarios del SNC no se estadifican con el sistema TNM.

Esta última actualización, conocida como "Clasificación de Tumores de la SNC de la OMS 2016", fue publicada en 2016 por el grupo de trabajo de la OMS compuesto por 35 neuropatólogos con la contribución de 117 expertos de 20 países y el apoyo de neurooncólogos y científicos de 10 países (Toro-Moreno et al.). La nueva clasificación OMS 2016 pretende una definición más completa de estas entidades, con un mejor estudio y conocimiento para prever un mejor manejo terapéutico. Por, tanto permite una clasificación de manera más objetiva y confiable (Mittler et al.).

Antes de la revisión OMS 2016, la gran mayoría de los gliomas infiltrantes se clasificaba en tres categorías: Astrocitomas, Oligoastrocitomas y Oligodendrogliomas (Johnson et al., 2017). Actualmente hay más de 100 tipos de tumores primarios del SNC, caracterizados por la histología y el perfil molecular, cada uno con su propio espectro de presentación clínica, tratamiento y pronóstico (Ostrom et al.). Por ello, existen organizaciones como la Central Brain Tumor Registry of the United States (CBTRUS) enfocadas exclusivamente en proporcionar datos estadísticos sobre tumores primarios del SNC. Probablemente presenta la mayor base de datos poblacional en histología de tumores primarios del SNC (Ostrom et al.). Otras bases de datos, son la Austrian Brain Tumor Registry (Wöhrer et al., 2009) (Austria) y la Swedish Brain Tumor Registry (Asklund et al., 2015) (Suecia). 


\section{MARCADORES MOLECULARES}

En consideración a los avances demostrados, la OMS ha presentado la clasificación nosológica 2016. En particular, las mutaciones de los genes IDH1/IDH2, la codelección $1 \mathrm{p} / 19 \mathrm{q}$ y las mutaciones en los genes que codifican las variantes de la histona $\mathrm{H} 3$ han cambiado drásticamente el conocimiento sobre estos tumores (Toro-Moreno et al.).

La introducción de estos marcadores moleculares otorga un componente objetivo al diagnóstico, con lo cual disminuye la variabilidad entre neuropatólogos (Okuma \& Fernández, 2017). Por ejemplo, el reconocimiento de mutaciones de IDH1 e IDH2 en altas frecuencias en astrocitomas y oligodendrogliomas de infiltración difusa, permite diferenciarlos y estratificarlos para definir manejo según pronóstico (Cardona et al., 2016). Un grupo de expertos neuropatólogos propuso el concepto del diagnóstico mediante capas, donde el diagnóstico final es aplicable sólo cuando se tiene todo el resto de capas (histología, grado OMS, información molecular). De esta forma cuando existen dudas histológicas se utilizaría la información molecular (Louis et al., 2014).

El proyecto piloto The Cancer Genome Atlas (TCGA) tiene como objetivo evaluar las características moleculares de los tumores para así otorgar una base de datos mundial (Chittenden et al., 2008). Un estudio empleando el TCGA evaluó 430 células de glioblastoma a través de la secuenciación de RNA y los clasificó en 4 grupos, diferenciándolos por su biología, pronóstico y terapia en proneural, neural, clásico y mesenquimal (Patel et al., 2014). De esta forma, se pudo definir que el glioblastoma proneural era la variante más frecuente y con mejor pronóstico, mientras que el gliobastoma mesenquimal es el de peor pronóstico (Verhaak et al., 2010).

\section{IMAGENOLOGÍA}

Durante las últimas décadas, las técnicas avanzadas de imagen en resonancia magnética (RM) han aumentado la precisión diagnóstica de esta modalidad de imagen para diferenciar los tumores cerebrales intraaxiales. Así lo demostró Yoon et al. (2018) en su estudio retrospectivo de casi 5.000 pacientes, donde confirmó que la precisión diagnóstica es estadísticamente superior con las técnicas avanzadas de RM sobre la RM convencional.

En este sentido, los biomarcadores de imagen se plantean como medidas objetivas de un proceso biológico o patológico específicos y sensibles, de fácil ejecución e inocuo, además de ser confiables, reproducibles y de bajo costo (Bonmatí et al., 2011). Sus mayores ventajas se centran en la ausencia de daño al paciente (no invasivos) y en la mejoría de la práctica clínica en estos tumores cerebrales al permitir la obtención óptima de información sobre sus características principales. La intención final es obtener un conocimiento complementario al que es posible alcanzar con la biopsia cerebral.

Las técnicas de RM potenciadas por perfusión (PWMRI) evalúan el flujo de sangre al tejido y la alteración de la barrera hematoencefálica. Usando el parénquima cerebral normal como referencia, estas técnicas pueden detectar las alteraciones de la vascularización del tejido y en la permeabilidad capilar vascular que ocurren debido a la angiogénesis aberrante inducida por el tumor (Nandu et al., 2018).

Las técnicas de PW-MRI pueden caracterizar los tejidos en función de las diferencias en el movimiento de las moléculas de agua libre. Así, se ha demostrado que la mayor celularidad y densidad celular del tumor se asocia con una disminución del coeficiente de difusión aparente (ADC), una medición calculada a partir de DW-MRI (Hayashida et al., 2006). Este parámetro permite distinguir entre gliomas de alto y bajo grado.

La espectroscopÍa de RM (MRS) mide las concentraciones de ciertos metabolitos dentro de los tejidos de manera no invasiva $(\mathrm{Oz}, 2007)$. Una limitación importante de la técnica es que es operador-dependiente, ya que requiere personal experimentado que seleccione manualmente las regiones de interés durante la adquisición y realice un procesado adecuado de los espectros obtenidos (Hui et al., 2008).

Con estos parámetros se han generado algoritmos que relacionan las carácterísticas imagenológicas con la biología del tumor. Esta correlación ha generado modelos computacionales, algunas con el uso de la Inteligencia Artificial, que han aportado datos estimativos sobre un diagnóstico más concreto, un pronóstico más preciso y una predicción de la respuesta al tratamiento más certera. Tal tecnología ha sido probada en otros cánceres sistémicos con éxito (Lambin et al., 2012) ; (Huang et al., 2016). Estas nuevas tecnologías permiten el acuñamiento de conceptos como el de biopsia virtual, en la cual la información obtenida por radiómica, genómica y metabolómica incorporará información de utilidad creciente el manejo oncológico (Sanduleanu et al., 2018).

\section{DISCUSIÓN}

Los avances en el diagnóstico de los Tumores (Primarios del SNC ) con el uso de tecnologías avanzadas, in- 
cluyendo la combinación de estudios anátomo-patológicos con los perfiles histopatológicos, moleculares y los biomarcadores de imagen, colaboran en conocer y relacionar con mayor especificidad las características de los distintos tipos de tumores. La profundización de estos conocimientos se estima que permitirá aún más definir los mejores protocolos de manejo terapéutico en estos pacientes.

Por ello, es necesario efectuar con más profundidad investigaciones clínicas que incorporen los diferentes marcadores moleculares y biomarcadores de imagen en pacientes con Tumores Primarios del SNC. Dentro de las limitaciones asociadas a los biomarcadores de imagen en la práctica asistencial, se puede considerar una difícil estandarización de los parámetros físicos de adquisición de las imágenes, el empleo de técnicas diferentes en el procesamiento de la información con modelamiento dinámico de la señal, y la disponibilidad de equipos de RM. Estas son algunas de las barreras que nos separan del establecimiento de criterios consensuados para la evaluación de pacientes con gliomas cerebrales (Okuma \& Fernández). Para solventar estas limitaciones es importante estandarizar tanto los parámetros físicos como las técnicas de procesamiento de información. De igual modo, es importante establecer criterios de estudio y de manejo según las características clínicas de los pacientes con Tumores Primarios del SNC.

Tales procesos permitirán comparar y validar los hallazgos imagenológicos encontrados, tanto en nuestro medio nacional como en el internacional, y así establecer una inferencia metodológica con la mejor batería de exámenes y protocolo a utilizar en estas lesiones.

Por tanto, corresponde a un desafío actual a nivel nacional generar un criterio consensuado y actualizado para desarrollar Guías Clínicas Chilenas, que permitan mejorar esta área de conocimiento y optimizar finalmente el manejo terapéutico.

El uso de la Inteligencia artificial y los macrodatos (Big Data) está evolucionando como una implementación práctica en salud, lo cual permite esperar una mejora en la atención al paciente. En Chile existen varios centros hospitalarios que dependen del Ministerio de Salud (MINSAL) y que poseen capacidades suficientes para estudiar con técnicas avanzadas de RM y de procesamiento de datos. De esta forma, se puede optimizar el uso de la tecnología disponible para aumentar el conocimiento y abordar con un manejo confiable y económico al paciente. Por tanto, tales estudios podrían aportar una valiosa información que permita generar protocolos estandarizados (basados en la evidencia) y así, construir modelos diagnósticos y predictivos, que nos permitan optimizar el uso de recursos para mejorar progresivamente el manejo de los pacientes.
GUAJARDO-HERNÁNDEZ, U.; GEISSE, M. F.; MARTÍBONMATÍ, L.; GEJMAN. E. R. \& ROJAS, Z. D. Molecular markers and image biomarkers. Importance in the therapeutic advance of intraaxial brain tumors.. Int. J. Morphol., 39(2):601606, 2021.

SUMMARY: The classification of primary tumors of the Central Nervous System (CNS) has its origin in the morphological description whose histopathological analysis has allowed to identify the cell line involved in these tumors and obtain the recognition of certain characteristics of these lesions and their clinical evolution. The molecular study has come to complement the initial diagnosis allowing to recognize entities that are not distinguishable in another way and that have varied the concepts and definitions of various pathological entities modifying the visible horizon of these diseases. The role of Magnetic Resonance (MR) images in the management of intraaxial tumors can be broadly divided into the diagnosis and classification of tumors, treatment planning and subsequent treatment. The present article summarizes the epidemiologic evidence related to the classification of primary tumors of the CNS with molecular markers and MR imaging biomarkers.

\section{KEY WORDS: Brain tumor, MR images; Biomarkers.}

\section{REFERENCIAS BIBLIOGRÁFICAS}

Asklund, T.; Malmström, A.; Bergqvist, M.; Björ, O. \& Henriksson, R. Brain tumors in Sweden: data from a population-based registry 1999 2012. Acta Oncol., 54(3):377-84, 2015.

Bonmatí, L. M.; Alberich-Bayarri, A.; García-Martía, G.; Requena, R. S.; Pérez Castillo, C.; Carot Sierrae, J. M. \& Manjón Herrera, J. V. Biomarcadores de imagen, imagen cuantitativa y bioingeniería. Radiología, 54(3):269-78, 2012.

Cardona, A. F.; Rojas, L.; Wills, B.; Behaine, J.; Jiménez, E.; Hakim, F.; Useche, N.; Bermúdez, S.; Arrieta, O.; Mejía, J. A.; Ramón, J. F.; Carranza, H.; Vargas, C.; Otero, J.; González, D.; Rodríguez, J.; Ortíz, L. D.; Cifuentes, H. \& Balaña, C. Genotyping low-grade gliomas among hispanics. Oncology Practice, 3(3):164-72, 2016.

Chittenden, T. W.; Howe, E. A.; Culhane, A. C.; Sultana, R.; Taylor, J. M.; Holmes, C. \& Quackenbush, J. Functional classification analysis of somatically mutated genes in human breast and colorectal cancers. Genomics, 91(6):508-11, 2008.

Cuevas, G. J. C.; Maya, G. I.; Villanueva, S. F. G.; Gaitán, C. L. A. \& Leyva, H. E. R. Estandarización en la observación y clasificación de lesiones epiteliales premalignas y malignas. Int. J. Morphol., 29(3):706-10, 2011.

Ferlay, J.; Soerjomataram, I.; Dikshit, R.; Eser, S.; Mathers, C.; Rebelo, M.; Parkin, D. M.; Forman, D. \& Bray, F. . Cancer incidence and mortality worldwide: sources, methods and major patterns in GLOBOCAN 2012. Int. J. Cancer, 1;136(5):E359-86, 2015.

Hayashida, Y.; Hirai, T.; Morishita, S.; Kitajima, M.; Murakami, R.; Korogi, Y.; Makino, K.; Nakamura, H.; Ikushima, I.; Yamura, M.; Kochi, M.; Kuratsu, J-i \& Yamashita, Y. Diffusion-weighted imaging of metastatic brain tumors: comparison with histologic type and tumor cellularity. AJNR Am. J. Neuroradiol., 27(7):1419-25, 2006.

Huang, Y.; Liang, C. H.; He, L.; Tian, J.; Liang, C-s.; Chen, X.; Ma, Z-I. \& Liu, Z-y. Development and Validation of a Radiomics Nomogram for Preoperative Prediction of Lymph Node Metastasis in Colorectal Cancer. J. Clin. Oncol., 34(18):2157-64, 2016. 
Hui, E. S.; Cheung, M. M.; Qi, L. \& Wu, E. X. Towards better MR characterization of neural tissues using directional diffusion kurtosis analysis. Neuroimage, 1;42(1):122-34, 2008.

Johnson, D. R.; Guerin, J. B.; Giannini, C.; Morris, J. M.; Eckel, L. J. \& Kaufmann, T. J. 2016 Updates to the WHO Brain Tumor Classification System: What the Radiologist Needs to Know. Radiographics, 37(7):2164-80, 2017.

Kleihues, P.; Louis, D. N.; Scheithauer, B. W.; Rorke, L. B.; Reifenberger, G.; Burger, P. C. \& Cavenee, W. K. The WHO Classification of Tumors of the Nervous System. J. Neuropathol. Exp. Neurol., 61(3):215-25, 2002.

Lambin, P.; Rios-Velazquez, E.; Leijenaar, R.; Carvalho, S.; van Stiphout, R. G. P. M.; Granton, P.; Zegers, C. M. L.; Gillies, R.; Boellard, R.; Dekker, A. \& Aerts, H. J. W. L. Radiomics: extracting more information from medical images using advanced feature analysis. Eur. J. Cancer, 48(4):441-6, 2012.

Louis, D. N.; Ohgaki, H.; Wiestler, O. D.; Cavenee, W. K.; Burger, P. C.; Jouvet, A.; Scheithauer, B. W. \& Kleihues, P. The 2007 WHO classification of tumours of the central nervous system. Acta Neuropathol., 114(2):97-109, 2007.

Louis, D. N.; Arie Perry, A.; Burger, P.; Ellison, D. W.; Guido Reifenberger, von Deimling, A.; Aldape, K.; Brat, D.; Collins, V. P.; Eberhart, C.; Figarella-Branger, D.; Fuller, G. N.; Giangaspero, F.; Giannini, C.; Hawkins, C.; Kleihues, P.; Korshunov, A.; Kros, J. M.; Lopes, M. B.; Ng, H. K.; Ohgaki, H.; Paulus, W.; Pietsch, T.; Rosenblum, M.; Rushing, E.; Soylemezoglu, F.; Wiestler, O.; Wesseling, P. \& International Society Of Neuropathology--Haarlem. International Society Of Neuropathology--Haarlem consensus guidelines for nervous system tumor classification and grading. Brain Pathol., 24(5):429-35, 2014.

Mittler, M. A.; Walters, B. C. \& Stopa, E. G. Observer reliability in histological grading of astrocytoma stereotactic biopsies. J. Neurosurg., 85(6): 1091-4, 1996.

Nandu, H.; Wen, P. Y \& Huang, R. Y. Imaging in neuro-oncology. Ther. Adv. Neurol. Disord., 2018. 11: 1756286418759865.

Okuma, C. \& Fernández, R. Evaluación de gliomas por técnicas avanzadas de resonancia magnética. Rev. Med. Clin. Condes, 28(3):360-77, 2017.

Ostrom, Q. T.; Gittleman, H.; Truitt, G.; Boscia, A.; Kruchko, C.; BarnholtzSloan, J. S. CBTRUS Statistical Report: Primary Brain and Other Central Nervous System Tumors Diagnosed in the United States in 20112015. Neuro. Oncol., 20(suppl. 4):1-86, 2018.

Oz, G.; Alger, J. R.; Barker, P. B.; Bartha, R.; Bizzi, A.; Boesch, C.; Bolan, P. J.; Brindle, K. M.; Cudalbu, C.; Dinçer, A.; Dydak, U.; Emir, U. E.; Frahm, J.; González, R. G.; Gruber, S.; Gruetter, R.; Gupta, R. K.; Heerschap, A.; Henning, A.; Hetherington, H. P.; Howe, F. A.; Hüppi, P. S.; Hurd, R. E.; Kantarci, K.; Klomp, D. W. J.; Kreis, R.; Kruiskamp, M. J.; Leach, M. O.; Lin, A. P.; Luijten, P. R.; Marjanska, M.; Maudsley, A. A.; Meyerhoff, D. J.; Mountford, C. E.; Nelson, S. J.; Pamir, M. N.; Pan, J. W.; Peet, A. C.; Poptani, H.; Posse, P.; Pouwels, P. J. W.; Ratai, E. M.; Ross, B. D.; Scheenen, T. W.; Schuster, C.; Smith, I. C. P.; Soher, B. J.; Tkác, I.; Vigneron, D. V.; Kauppinen, R. A.; MRS Consensus Group. Clinical proton MR spectroscopy in central nervous system disorders. Radiology, 270(3):658-79, 2014

Patel, A. P.; Tirosh, I.; Trombetta, J. J.; Shalek, A. K.; Gillespie, S. M.; Wakimoto, H.; Cahill, D. P.; Nahed, B. V.; Curry, W. T.; Martuza, R. L.; Louis, D. N.; Rozenblatt-Rosen, O.; Suvà, M. L.; Regev, A. \& Bernstein, B. E. Single-cell RNA-seq highlights intratumoral heterogeneity in primary glioblastoma. Science, 20:344(6190):1396-401, 2014.

Sanduleanu, S.; Woodruff, H. C.; Jong, E. E. C.; van Timmeren, J. E.; Jochems, A.; Dubois L. \& Lambin, P. Tracking tumor biology with radiomics: A systematic review utilizing a radiomics quality score. Radiother. Oncol., 127(3):349-60, 2018.

Toro-Moreno, A. C.; Srna-Vélez, L.; Gallego-González, D.; JaramilloJaramillo, L. I.; Martñínez-Sánchez, L. M. \& Álvarez-Hernández, L. F. Tumores de sistema nervioso central en pediatría: Presente y futuro del abordaje diagnóstico. Rev. Ecuatoriana Neurol. 26(3):283-8, 2017.

Verhaak, R. G. W.; Hoadley, K. A.; Purdom, E.; Wang, V.; Qi, Y.; Wilkerson, M. D.; Miller, C. R.; Ding, L.; Golub, T.; Mesirov, J. P.; Alexe, G.;
Lawrence, M.; O'Kelly, M.; Tamayo, P.; Weir, B. A.; Gabrie, S.; Winckler, W.; Gupta, S.; Jakkula, L.; Feiler, H. S.; Hodgson, J. H.; James, C. D.; Sarkaria, J. N.; Brennan, C.; Kahn, A.; Spellman, P. T.; Wilson, R. K.; Speed, T. P.; Gray, J. W.; Meyerson, M.; Getz, G.; Perou, C. M.; Hayes, D. N. \& The Cancer Genome Atlas Research Network. An integrated genomic analysis identifies clinically relevant subtypes of glioblastoma characterized by abnormalities in PDGFRA, IDH1, EGFR and NF1. Cancer Cell, 19:17(1): 98- 1102010.

Wöhrer, A.; Waldhör, T.; Heinz, H.; Hack, M.; Feichtinger, J.; GruberMösenbacher, U.; Kiefer, A.; Maier, H.; Motz, R.; Reiner-Concin, A.; Richling, B.; Idriceanu, C.; Scarpatetti, M.; Sedivy, R.; Bankl, H. C.; Stiglbauer, W.; Preusser, P.; Rössler, K. \& Hainfellner, J. A. The Austrian Brain Tumour Registry: a cooperative way to establish a populationbased brain tumour registry. J. Neurooncol., 95(3):401-11, 2009.

Yoon, R. A.; Kim, H. S.; Hong, G. S.; Park, J. E.; Jung, S. C. \& Kim J. H. Joint approach of diffusion- and perfusion-weighted MRI in intra-axial mass like lesions in clinical practice simulation. PLoS One, 13(9): e0202891, 2018.

Zülch, K. J. Histological typing of tumours of the central nervous system. Geneva, World Health Organization. International histological classification of tumours, 21, 1979.

\section{Dirección para correspondencia: \\ Ulises Guajardo Hernández}

Departamento de Especialidades Médicas

Facultad de Medicina

Universidad de La Frontera

Temuco

CHILE

E-mail: ulises.guajardo@ufrontera.cl

Recibido: 09-09-2020

Aceptado: 16-01-2021 